\title{
HEALING POTENTIAL OF IMMATURE PERMANENT TEETH WITH NECROTIC PULPS AND PERIAPICAL RADIOLUCENCY
}

\author{
Akram Elsayyad*, Manal Ahmed Elsayed* and Kamal Elmotayam*
}

\begin{abstract}
Introduction: Regenerative endodontics is a promising alternative treatment for immature teeth with necrotic pulps. The present study was performed to assess periapical healing potential of young permanent immature teeth with necrotic pulp after the following treatment protocols: (1) Calcium hydroxide $\mathrm{Ca}(\mathrm{OH})^{2}$ apexification (2) the regenerative endodontic protocol (blood clot scaffold).

Methods: Immature necrotic permanent maxillary central incisors $(n=48)$ were divided into 2 groups according to the treatment protocol; Group A: the apexification group and Group B: the REG group (regenerative endodontic protocol). Standardized radiographs were digitally evaluated for healing of periapical radiolucency. Results: After a follow-up period of 18 months, most of the cases showed radiographic evidence of periapical healing.
\end{abstract}

Results: After a follow-up period of 18 months, most of the cases showed radiographic evidence of periapical healing.

Conclusions: The regenerative endodontic procedure allowed better periapical healing in teeth with necrotic pulps and periapical radiolucency.

KEY WORDS : Mineral trioxide aggregate, Regeneration, Apexification.

\section{INTRODUCTION}

Loss of pulp vitality in anterior permanent teeth of children was more commonly attributed to traumatic injuries than to any other causes. This high incidence of traumatic injuries is responsible for the greater frequency with which periapical pathological conditions develop in those teeth. If the dental pulp is damaged before development of the root length and closure of the apical foramen, normal root development is altered or halted completely ${ }^{(1)}$.

Such conditions present difficulty in root canal debridement and obturation because of the open apex. Such cases were traditionally treated by apexification procedures using calcium hydroxide. Such management requires long-term placement of

* Department of Pediatric dentistry and Dental Public Heath, Faculty of Dentistry, Cairo University. 
calcium hydroxide inside the root canal to induce the formation of an apical hard tissue barrier. Periapical tissues in immature teeth are rich in blood supply and contain stem cells that have the potentiality for tissue regeneration. Under suitable conditions, stem cells can be programmed for self-regeneration to restore the lost part. Hence, the concept of regeneration of immature nonvital teeth was advocated (2,3) Eradication of bacteria from the canal space is mandatory for successful regenerative endodontic procedures. Research with topical antibiotics showed that a combination of metronidazole, and ciprofloxacin could be effective against common endodontic pathogens ${ }^{(4)}$.

We are living in the age of evidence-based medicine. Any new concepts and techniques to be used in the clinic should ideally be assessed in randomized controlled clinical trials against their respective gold standards. Randomized clinical trials comparing the long-term success of this new revascularization treatment with traditional ones, especially apexification and MTA apical plug techniques, have not been performed. In addition, the criteria for case selection and criteria of success/ failure have not been determined ${ }^{(5)}$. Moreover, most treatments used and described in successful revascularization cases so far are different from each other. The aim of the present investigation was to evaluate clinically and radiographically the effect of two different materials on periapical healing process of traumatized immature non-vital permanent anterior teeth in children.

When abnormal development of the root occurs, apexification treatment must be initiated. The traditional treatment of immature teeth with necrotic pulps and apical periodontitis involves longterm application of calcium hydroxide to induce apexification. A new regenerative/revascularization treatment option for immature permanent teeth with apical periodontitis has been introduced. It involves disinfecting the root canal system, providing a matrix of blood clot into which cells could grow, and sealing of the coronal access. This treatment approach offers clinicians a great potential to avoid the need for traditional apexification with calcium hydroxide or the need to achieve an artificial apical barrier with mineral trioxide aggregate. Furthermore, this treatment approach can help rescue infected immature teeth by physiologically strengthening the root walls ${ }^{(6)}$.

\section{MATERIALS AND METHODS}

Forty-eight immature, nonvital maxillary anterior teeth presenting with or without signs and/ or symptoms of periapical pathology were included in this study from the outpatient clinic of the Faculty of Dentistry, Cairo University. A detailed medical and dental history was obtained from each patient's parents or guardians. Children were apparently healthy and had no congenital or systemic chronic disease. Intraoral periapical radiographs revealed immature apices. The age of the patients ranged between 9 and 13 years. Informed consent was signed for each case by the patient's parents or guardians including the proposed treatment and possible outcomes or complications. The selected teeth were divided randomly into two main groups according to the technique used: Group A: Consisted of 26 teeth that were treated with regeneration (revascularization) technique.

Group B: Consisted of 22 teeth that were treated with apexification technique.

Preoperative radiographs were taken using the standardized paralleling technique with the Rinn XCP alignment system preoperatively using standardized periapical radiographs and specially designed DigoraÒ software for image analysis.

After local anesthesia and rubber dam isolation. A conventional access cavity was prepared in the palatal surface of the teeth using carbide burs. The root canal was irrigated copiously with $5.25 \%$ sodium hypochlorite $(\mathrm{NaOCl})$ using a hypodermic 
syringe. The canal space was dried using paper points. A sterile cotton pellet was then applied, and the access cavity was sealed using a temporary restoration for 2 weeks. The final visit was scheduled when the tooth was asymptomatic with no signs of discharge. After anesthesia and proper isolation, the temporary restoration and the cotton pellet were removed. The canal was irrigated with $10 \mathrm{~mL} \mathrm{NaOCl}$ $5.25 \%$ followed by $10 \mathrm{~mL}$ sterile saline and dried with sterile paper points. One of the following treatment modalities was randomly chosen.

\section{For Group A}

Antimicrobial agents consisting of equal parts of metronidazol and ciprofloxacin in a paste form as intra-canal medication was applied using lentulo spiral. The access cavity was sealed with a cotton pellet and $2 \mathrm{~mm}$-thick temporary filling. After 1 week a sterile hand file size \#80 was used with sharp strokes into the periapical tissue $2 \mathrm{~mm}$ beyond the apex until bleeding was evident at the cervical portion of the canal. An MTA orifice plug was used to seal the canal orifice covered by a moist cotton pellet for subgroup I. while in subgroup II Calcium Hydroxide $\mathrm{Ca}(\mathrm{OH})_{2}$ paste was prepared by mixing $\mathrm{Ca}(\mathrm{OH})_{2}$ powder with distilled water and rolled to form a robe that was inserted in the canal, followed by a thread of cotton and hand plugger condensation over the blood clot. After 1 week, adhesive composite resin was used to seal the access cavity.

\section{For Group B}

The MTA apical plugs were placed by a standardized hand condensation technique of apical stop in all cases of Subgroup I. The thicknesses of the MTA apical plug varied from $3 \mathrm{~mm}$ to $5 \mathrm{~mm}$, which offers a good seal and allow the rest of the canal to be obturated by using thermoplasticized gutta purcha. The patient was instructed to wait in the waiting area for one hour to allow for the formation a firm barrier of MTA cement, which was assessed using a $\mathrm{K}$ File. The rest of the canal was backfilled with injectable thermoplasticized gutta percha which offers a hermetic seal to the canal using E\&QÒ Cordless Gutta Percha Obturation Systems. While For Subgroup II Calcium Hydroxide $\mathrm{Ca}(\mathrm{OH})_{2}$ paste was prepared by mixing $\mathrm{Ca}(\mathrm{OH})_{2}$ powder with distilled water and rolled to form a robe that was inserted in the canal followed by a thread of cotton and endodontic plugger condensation until $\mathrm{Ca}(\mathrm{OH})_{2}$ reaches the end of canal. The access cavity was then sealed with glass ionomer and adhesive composite resin.

\section{Evaluation}

Patients were recalled at $3,6,12$, and 18 months. Follow-up included the radiographic assessment for apical closure, root maturation and pulpal canal obliteration. Images of the treated teeth were manipulated using specially designed software of the DigoraÒ system.

\section{RESULTS}

Table (1) show frequencies, percentages and results of chi-square test for comparison between clinical success and failure in Group A and Group B. There was no statistically significant difference between clinical outcomes in the two main groups $(\mathrm{P}$ value $=0.272)$. However, Group A showed higher clinical success (100\%) than Group B (95.5\%).

TABLE (1): Frequencies, percentages and results of chi-square test for comparison between clinical outcomes in Group A and Group B.

\begin{tabular}{|c|c|c|c|}
\hline $\begin{array}{c}\text { Clinical } \\
\text { outcome }\end{array}$ & $\begin{array}{c}\text { Group A } \\
(\mathrm{n}=26)\end{array}$ & $\begin{array}{c}\text { Group B } \\
(\mathrm{n}=22)\end{array}$ & $P$-value \\
\hline Success & $26(100 \%)$ & $21(95.5 \%)$ & \multirow{2}{*}{0.272} \\
\cline { 1 - 2 } Failure & $0(0 \%)$ & $1(4.5 \%)$ & \\
\hline
\end{tabular}

*: Significant at $P \leq 0.05$ 
Table (2) show frequencies, percentages and results of chi-square test for comparison between radiographic success and failure in Group A and Group B. There was no statistically significant difference between radiographic outcomes in the two main groups ( $\mathrm{p}$ value $=0.502$ ). However, Group A showed higher radiographic success $(92.3 \%)$ compared to Group B (86.4\%).

TABLE (2): Frequencies, percentages and results of chi-square test for comparison between radiographic findings in Group A and Group B.

\begin{tabular}{|c|c|c|c|}
\hline $\begin{array}{c}\text { Radiographic } \\
\text { outcome }\end{array}$ & $\begin{array}{c}\text { Group A } \\
(\mathrm{n}=26 \text { teeth })\end{array}$ & $\begin{array}{c}\text { Group B } \\
(\mathrm{n}=22 \text { teeth })\end{array}$ & \multirow{2}{*}{$P$-value } \\
\hline Success & $24(92.3 \%)$ & $19(86.4 \%)$ & \multirow{2}{*}{0.502} \\
\cline { 1 - 2 } Failure & $2(7.7 \%)$ & $3(13.6 \%)$ & \\
\hline
\end{tabular}

*: Significant at $P \leq 0.05$

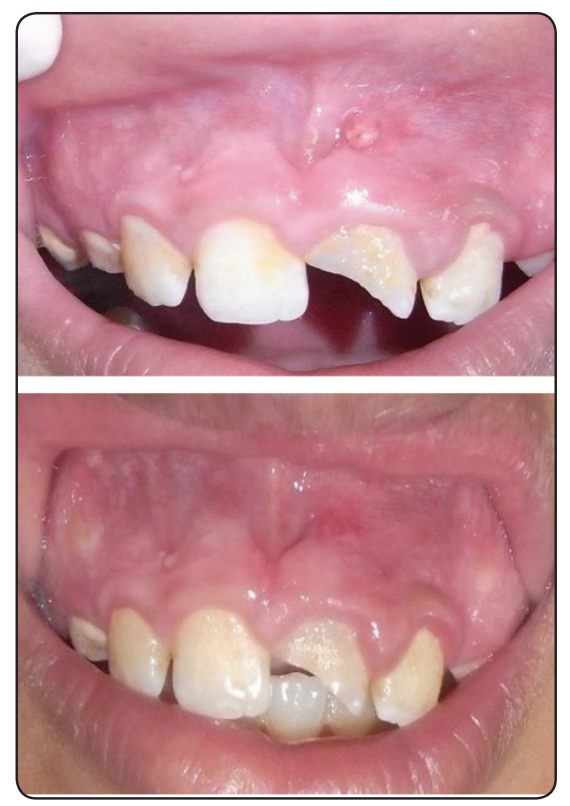

Fig. (1): Clinical success (healing of buccal sinus).

\section{DISCUSSION}

Necrosis of a dental pulp can be either infected or noninfected. In the event of necrosis and canal infection of immature teeth, it is necessary to disinfect the root canal and stimulate the formation of an apical barrier that would allow an adequate filling, i.e. an apexification procedure. Different techniques for apexification have been suggested, the most common being calcium hydroxide for an undetermined period of time ${ }^{(7,8,9)}$.

It is clear that there is some urgency to establish the most predictable protocol to treat such cases. Hence, the present study was conducted to evaluate the efficacy of revascularization procedures in the healing process of the periapical tissues using the regenerative potential of young permanent immature teeth with necrotic infected pulps following revascularization by blood clot and apexification including regular follow-up appointments to assess the treatment response in terms of clinical and radiographic healing.

The current study also compared the clinical success of revascularization and apexification techniques collectively (Group A and Group B, respectively). There was no statistically significant difference between clinical success in the two main groups. However, revascularization group showed $100 \%$ clinical success rate compared to apexification group which showed $95.5 \%$ clinical success rate where one $\mathrm{Ca}(\mathrm{OH})_{2}$ apexification case failed to achieve criteria of clinical success. This failure may be due to the fact that calcium hydroxide was washed out by tissue fluids through the open apex leaving the canal susceptible to reinfection according to Trope, (2006). This result was confirmed by Thibodeau et al., (2007), and Lenzi and Trope, (2012) who reported complete resolution of symptoms, with positive response to pulp testing following 1 year of regeneration of immature teeth with apical periodontitis. This result was disagreed by Felippe et al., (2005) who revealed that instrumentation and irrigation with 
sodium hypochlorite did not disinfect the canals and reported absence of apical calcified tissue barrier, presence of a severe inflammatory reaction, bone and root resorption and microorganisms. Nevertheless, this result of high clinical success of revascularization technique was not confirmed by Ding et al., (2009), who reported continuation of symptoms, after using the same disinfection procedure advocated in this study with $\mathrm{NaOCl}$ $5.25 \%$ and triple antibiotic dressing, in 2 patients out of 12 that caused changes in treatment plan from revascularization to apexification.

The current study compared also the radiographic success between revascularization and apexification groups. There was no statistically significant difference between radiographic success in the two groups. However, revascularization group showed higher radiographic success $(92.3 \%)$ compared to apexification group $(86.4 \%)$. This result was agreed by Avinash et al., (2006) who concluded that apexification using $\mathrm{Ca}(\mathrm{OH})_{2}$ may not be effective on large periapical lesions. This result could be explained on the basis that during apexification procedure the root canal is susceptible to reinfection because it is covered by a temporary filling, which doesn't provide adequate seal (Leonardo, 1993). However, this result was disagreed by Villa and Ferna'ndez, (2005) who observed complete healing of the periradicular lesion of immature teeth after filling the root canal with calcium hydroxide for a period of 8 months. Moreover, Kalaskar et al., (2004) observed complete healing of large periapical lesions and apical closure of wide-open apex and of pulpless teeth using only calcium hydroxide paste. Oztan (2002) used calcium hydroxide paste as an intracanal medicament in which large periapical lesions healed completely within a period of 3 months. Lenzi and Trope, (2012) demonstrated healing of periapical lesion and production of hard-tissue barrier in the apex after regenerative endodontic treatment even in the absence of vital tissue inside the root canal space. They concluded that disinfection protocol in regenerative endodontic treatments $(\mathrm{NaOCl}$ irrigation followed by 1 month of triple antibiotic dressing) renders the root canal space sterile or has a long-lasting effect suppressing the regrowth of bacteria over time.

\section{CONCLUSIONS}

1. Revascularization is an effective technique for managing immature permanent teeth with apical periodontitis with appropriate case selection.

2. Revascularization technique offers several advantages over other established treatment protocols for the management of immature teeth with infected root canals.

3. The key factor for the success of revascularization process in necrotic, infected, immature teeth is disinfection of the root canal system.

4. The use of triple antibiotic paste for disinfection of root canals of immature non-vital teeth should be advocated.

\section{REFERENCES}

1. Holden DT, Schwartz SA, Kirkpatrick TC and Schindler WG. Clinical Outcomes of Artificial Root-end Barriers with Mineral Trioxide Aggregate in Teeth with Immature Apices. JOE, 2008; 34: 812-7.

2. Iwaya SI, Ikawa M, Kubota M. Revascularization of an immature permanent tooth with apical periodontitis and sinus tract. Dent Traumatol 2001;17:185-7.

3. Banchs F, Trope M. Revascularization of immature permanent teeth with apical periodontitis: new treatment protocol? J Endod, 2004; 30:196-200.

4. Chueh L and Huang G. Immature teeth with periradicular periodontitis or abscess undergoing apexogenesis: a paradigm shift. J Endod 2006; 32: 1205-13.

5. Nosrat A, Negar H, and Kaveh O. Drawbacks and Unfavorable Outcomes of Regenerative Endodontic Treatments of Necrotic Immature Teeth: A Literature Review and Report of a Case. JOE (38); 10: October 2012.

6. Thibodeau B, Trope M. Pulp revascularization of a necrotic infected immature permanent tooth: case report and review of the literature. Pediatric Dentistry 2007; 29: 47-50. 
7. EstrelaC, Pimenta FC, I to IY and Bammann LL. In vitro determination of direct antimicrobial effect of calcium hydroxide. JOE, 2001; 24: 15-7.

8. Villa P, Ferna'ndez R. Apexification of a replanted tooth using mineral trioxide aggregate. Dent Traumatol 2005; 21: 306-308._Blackwell Munksgaard, 2005.

9. Rafter M. Apexification: a review. Dent Traumatol 2005; $21: 1-8$.

10. Ding RY, Cheung GS, Chen J, Yin XZ, Wang QQ, Zhang CF. Pulp revascularization of immature teeth with apical periodontitis: a clinical study. J Endod 2009; 35:745-9.

11. Avinash RS, Manoj GC and Manwar NU Blunder Buss Canal: a challenge for endodontist. Case Report. Textbook of Endodontology; 77-81, 2006.
12. Felippe WT, Felippe MC, Rocha MJ. The effect of mineral trioxide aggregate on the apexification and periapical healing of teeth with incomplete root formation. Int Endod J, 2006; 39: 2-9.

13. Lenzi R and Trope M. Revitalization procedures in two traumatized incisors with different biological outcomes. J Endod 2012; 38: 411-4.

14. Trope M. Treatment of the Immature Tooth with a Non -Vital Pulp and Apical Periodontitis. Endodontic Topics, 2006; 14: 51-9.

15. Leonardo M. Histological valuation of therapy using a calcium hydroxide dressing for teeth with incompletely formed apices and periapical lesion. J Endod, 1993; 19: 348-52. 